\title{
NONCOMMUTATIVE JORDAN ALGEBRAS OF CAPACITY TWO ${ }^{(1)}$
}

\author{
BY \\ KIRBY C. SMITH
}

\begin{abstract}
Let $J$ be a noncommutative Jordan algebra with 1. If $J$ has two orthogonal idempotents $e$ and $f$ such that $1=e+f$ and such that the Peirce 1-spaces of each are Jordan division rings, then $J$ is said to have capacity two. We prove that a simple noncommutative Jordan algebra of capacity two is either a Jordan matrix algebra, a quasi-associative algebra, or a type of quadratic algebra whose plus algebra is a Jordan algebra determined by a nondegenerate symmetric bilinear form.
\end{abstract}

We refer the reader to [1] for all the basic concepts used in this paper as well as for all definitions of terms with the exception of quasi-associative algebra which may be found in [3].

Introduction. Recently much work has been done on the structure of simple noncommutative Jordan algebras $J$ over a field $\Phi$ of characteristic not 2 which satisfy the following axioms:

(i) $J$ has an identity element 1 .

(ii) $1=e_{1}+e_{2}+\cdots+e_{n}$, where the $e_{i}$ 's are mutually orthogonal idempotents.

(iii) The Peirce 1-space with respect to $e_{i}, J_{1}\left(e_{i}\right)$, is a Jordan division ring for $i=1,2, \ldots, n$.

An algebra satisfying axioms (i)-(iii) is said to have capacity $n$.

N. Jacobson [2] proved that those simple commutative Jordan algebras of capacity $n$, where $n \geqq 3$, are Jordan matrix algebras. K. McCrimmon and R. D. Schafer in [5] proved that the simple, not commutative Jordan algebras of capacity $n(n \geqq 3)$ are all quasi-associative. In [6] J. M. Osborn found that a simple Jordan algebra of capacity two is either a $2 \times 2$ Jordan matrix algebra or a Jordan algebra determined by a nondegenerate symmetric bilinear form. It is the goal of this paper to characterize the simple noncommutative Jordan algebras of capacity two.

For any idempotent $e$ in a noncommutative Jordan algebra $J$ we have the Peirce decomposition of $J$ with respect to $e$; namely $J=J_{0}(e)+J_{1 / 2}(e)+J_{1}(e)$ where $J_{i}=\left\{x_{i} \mid \frac{1}{2}\left(e x_{i}+x_{i} e\right)=i x_{i}\right\}$. Throughout this work $J$ will denote a noncommutative

Presented to the Society, January 22, 1970; received by the editors October 7, 1970.

AMS 1969 subject classifications. Primary 1740.

Key words and phrases. Simple noncommutative Jordan algebra, capacity two, quasiassociative algebra.

(1) Research was supported in part by National Science Foundation contracts GP-3993 and GP-7235. This work is part of the author's doctoral dissertation at The University of Wisconsin under Professor J. Marshall Osborn.

Copyright (C) 1971, American Mathematical Society 
Jordan algebra of capacity two and $e$ and $f$ will be the two orthogonal idempotents in $J$ such that $1=e+f$ and $J_{0}$ (with respect to $e$ ) and $J_{1}$ are Jordan division rings. If $J_{1 / 2} \neq\{0\}$, which will be the case if $J$ is simple, then it is not hard to show that any Jordan ring of capacity two may be considered an algebra of capacity two over an appropriate field.

1. Osborn's results. If $J=J_{1}+J_{1 / 2}+J_{0}$ is simple then $J^{+}=J_{1}^{+}+J_{1 / 2}^{+}+J_{0}^{+}$will be a simple Jordan algebra of capacity two [5, p. 2]. From Osborn's work [6] we know that if $J^{+}$is not a Jordan algebra determined by a symmetric bilinear form, then $J^{+}$is a Jordan matrix algebra $H\left(E_{2}, \gamma\right)$, the set of symmetric $2 \times 2$ matrices under the canonical involution determined by the invertible diagonal matrix $\gamma$ and where $E$ is the associative algebra with involution * generated by

$$
\begin{array}{r}
S=\left\{2 \bar{R}_{x_{1}}^{+} \mid x_{1} \in J_{1}^{+} \text {and } \bar{R}_{x_{1}}^{+} \in \operatorname{Hom}_{\Phi}\left(J_{1 / 2}, J_{1 / 2}\right)\right. \\
\text { defined by } \left.\bar{R}_{x_{1}}^{+}\left(y_{1 / 2}\right)=\frac{1}{2}\left(x_{1} y_{1 / 2}+y_{1 / 2} x_{1}\right)\right\} .
\end{array}
$$

The nonzero generators of $E$ are symmetric and invertible and moreover every symmetric element of $E$ is a generator. $E$ is one of the following two types:

(a) An associative division algebra with involution which is not commutative and which is not a quaternion algebra over its center with the standard involution.

(b) A direct sum of a division algebra (which is not commutative) with its antiisomorphic copy and the involution switches components.

Finally $\gamma=\left(\begin{array}{cc}\gamma_{1} & 0 \\ 0 & \gamma_{2}\end{array}\right)$ where each $\gamma_{i}$ is a symmetric, invertible element of $E$.

If $\gamma_{1} \neq 1$ we change the involution in $E$ to be $\bar{\alpha}=\gamma_{1}^{-1} \alpha^{*} \gamma_{1}$ where $*$ is the old involution on $E$ and at the same time we change $\gamma$ to be $\left(\begin{array}{cc}1 & 0 \\ 0 & \gamma_{1}^{-1} \gamma_{2}\end{array}\right)$. Under the new involution, $\gamma_{1}^{-1} \gamma_{2}$ is symmetric and invertible. An easy calculation shows that using the new involution on $E$ and the new involution on $E_{2}$ determined by the new $\gamma$, the set $H\left(E_{2}, \gamma\right)$ remains the same as before. So we may assume that $\gamma$ is of the form $\left(\begin{array}{cc}1 & 0 \\ 0 & \gamma_{2}\end{array}\right)$ in $H\left(E_{2}, \gamma\right)$ and this we will do for the remainder of this work. We note that if $(E, *)$ is of type (a) or (b) then $(E,-)$ will be also.

Products in $J$ as well as those in $E$ will be denoted by juxtaposition.

2. Preliminary results. We define $\bar{R}_{x_{1}} \in \operatorname{Hom}_{\Phi}\left(J_{1 / 2}, J_{1 / 2}\right)$ by

$$
\bar{R}_{x_{1}}\left(y_{1 / 2}\right)=\left[y_{1 / 2} x_{1}\right]_{1 / 2} \text {, }
$$

i.e. $\bar{R}_{x_{1}}\left(y_{1 / 2}\right)$ is the component of $y_{1 / 2} x_{1}$ in $J_{1 / 2}$. (Likewise we define $\bar{L}_{x_{1}}\left(y_{1 / 2}\right)$ $=\left[x_{1} y_{1 / 2}\right]_{1 / 2}$. $)$ Then $2 \bar{R}_{x_{1}}^{+}=\bar{R}_{x_{1}}+\bar{L}_{x_{1}}$ and $E$ is generated by $\left\{\left(\bar{R}_{x_{1}}+\bar{L}_{x_{1}}\right) \mid x_{1} \in J_{1}\right\}$. Note that the identity 1 of $E$ is $2 \bar{R}_{e}^{+}=\bar{R}_{e}+\bar{L}_{e}$.

$J_{1 / 2}$ is a left $E$-module by

$$
\left(\bar{R}_{x_{1}}+\bar{L}_{x_{1}}\right) a_{1 / 2}=a_{1 / 2} x_{1}+x_{1} a_{1 / 2}
$$

where $a_{1 / 2} \in J_{1 / 2} . J_{1 / 2}$ as an $E$-module is isomorphic to $E$ as an $E$-module. Since $\operatorname{Hom}_{E}(E, E)$ is anti-isomorphic to $E$, so is $\operatorname{Hom}_{E}\left(J_{1 / 2}, J_{1 / 2}\right)$. We know that $E_{x} F_{e}=F_{e} E_{x}$ if $x \in J_{0}+J_{1}$ and $E, F=R, L\left[4\right.$, p. 188] and so $\bar{R}_{e}$ and $\bar{L}_{e}$ are in the center of $\operatorname{Hom}_{E}\left(J_{1 / 2}, J_{1 / 2}\right)$ and can be regarded as elements in the center of $E$. 
K. McCrimmon $[4$, p. 189] has shown that

$$
\begin{gathered}
\bar{L}_{e}\left(\bar{L}_{y_{1}}+\bar{R}_{y_{1}}\right)=\bar{L}_{y_{1}}, \\
\bar{R}_{e}\left(\bar{L}_{y_{1}}+\bar{R}_{y_{1}}\right)=\bar{R}_{y_{1}} ; \\
\bar{L}_{x_{1} y_{1}}=\bar{L}_{x_{1}} \bar{L}_{y_{1}}+\bar{L}_{y_{1}} \bar{R}_{x_{1}}, \\
\bar{R}_{x_{1} y_{1}}=\bar{R}_{x_{1}} \bar{L}_{y_{1}}+\bar{R}_{y_{1}} \bar{R}_{x_{1}} .
\end{gathered}
$$

The equations of (1) show that $\bar{L}_{y_{1}}$ and $\bar{R}_{y_{1}}$ are in $E$ for every $y_{1} \in J_{1}$.

Define $\nu: J_{1} \rightarrow S$ by $\nu\left(x_{1}\right)=\bar{R}_{x_{1}}+\bar{L}_{x_{1}} . \nu$ is onto and one-to-one. Using (1) and (2) we have

$$
\begin{aligned}
\bar{L}_{e} \nu\left(x_{1}\right) \nu\left(y_{1}\right)+\bar{R}_{e} \nu\left(y_{1}\right) \nu\left(x_{1}\right) & =\bar{L}_{e}\left(\bar{R}_{x_{1}}+\bar{L}_{x_{1}}\right)\left(\bar{R}_{y_{1}}+\bar{L}_{y_{1}}\right)+\bar{R}_{e}\left(\bar{R}_{y_{1}}+\bar{L}_{y_{1}}\right)\left(\bar{R}_{x_{1}}+\bar{L}_{x_{1}}\right) \\
& =\left(\bar{R}_{x_{1}}+\bar{L}_{x_{1}}\right) \bar{L}_{e}\left(\bar{R}_{y_{1}}+\bar{L}_{y_{1}}\right)+\left(\bar{R}_{y_{1}}+\bar{L}_{y_{1}}\right) \bar{R}_{e}\left(\bar{R}_{x_{1}}+\bar{L}_{x_{1}}\right) \\
& =\left(\bar{R}_{x_{1}}+\bar{L}_{x_{1}}\right) \bar{L}_{y_{1}}+\left(\bar{R}_{y_{1}}+\bar{L}_{y_{1}}\right) \bar{R}_{x_{1}} \\
& =\bar{L}_{x_{1} y_{1}}+\bar{R}_{x_{1} y_{1}}=\nu\left(x_{1} y_{1}\right) .
\end{aligned}
$$

Thus,

$$
\nu\left(x_{1} y_{1}\right)=\bar{L}_{e} \nu\left(x_{1}\right) \nu\left(y_{1}\right)+\bar{R}_{e} \nu\left(y_{1}\right) \nu\left(x_{1}\right) .
$$

We are now assuming that $J^{+}$is isomorphic to $H\left(E_{2}, \gamma\right)$ and so we can consider $J_{0}, J_{1 / 2}, J_{1}$ as sets of matrices of the form $\left(\begin{array}{cc}0 & 0 \\ 0 & \alpha\end{array}\right),\left(\begin{array}{cc}0 \\ \gamma_{2}^{-1} \delta^{*} & 0 \\ 0\end{array}\right),\left(\begin{array}{cc}\beta & 0 \\ 0 & 0\end{array}\right)$, respectively, where $\alpha, \delta \in E$ with $\alpha$ having the property that $\gamma_{2}^{-1} \alpha^{*} \gamma_{2}=\alpha$ and where

$$
\beta \in S=\left\{\beta \in E \mid \beta^{*}=\beta\right\}=\left\{\left(\bar{R}_{x_{1}}+\bar{L}_{x_{1}}\right) \mid x_{1} \in J_{1}\right\} .
$$

We are ready to describe the multiplication in $J_{1}$. For notation we will let $\alpha e$ denote the element $\left(\begin{array}{ll}\alpha & 0 \\ 0 & 0\end{array}\right)$ in $J_{1}$. If $x_{1}=\alpha e$ and $y_{1}=\beta e$, then (3) implies $(\alpha e)(\beta e)$ $=\left(\bar{L}_{e} \alpha \beta+\bar{R}_{e} \beta \alpha\right) e$ where $\bar{L}_{e} \alpha \beta+\bar{R}_{e} \beta \alpha \in S$. We have therefore proved

Lemma 1. If $\alpha e, \beta e \in J_{1}$, then $(\alpha e)(\beta e)=\left(\bar{L}_{e} \alpha \beta+\bar{R}_{e} \beta \alpha\right) e$.

LEMMA 2. The involution * on $E$ has the property that $\bar{L}_{x_{1}}^{*}=\bar{R}_{x_{1}}$ for every $x_{1} \in J_{1}$.

Proof. From Lemma 1 we know that

$$
\left(\bar{L}_{e} \alpha \beta+\bar{R}_{e} \beta \alpha\right)^{*}=\bar{L}_{e} \alpha \beta+\bar{R}_{e} \beta \alpha .
$$

Since $\bar{L}_{e}+\bar{R}_{e}=1$, (4) implies

$$
\alpha \beta-\beta \alpha=\left(\bar{L}_{e}^{*}+\bar{L}_{e}\right)(\alpha \beta-\beta \alpha)
$$

or

$$
\left[1-\left(\bar{L}_{e}^{*}+\bar{L}_{e}\right)\right](\alpha \beta-\beta \alpha)=0,
$$

for every $\alpha, \beta \in S$. Since $E$ is not commutative there exists $\alpha, \beta \in S$ such that $\alpha \beta-\beta \alpha$ $\neq 0$ and since $1-\left(\bar{L}_{e}^{*}+\bar{L}_{e}\right) \in S$ it is either 0 or invertible. So from the above equation we conclude that $1-\left(\bar{L}_{e}^{*}+\bar{L}_{e}\right)=0$ and this means $\bar{L}_{e}^{*}+\bar{L}_{e}=1$ and hence $\bar{L}_{e}^{*}=\bar{R}_{e}$. Using the equations of (1) we get $\bar{L}_{x_{1}}^{*}=\bar{R}_{x_{1}}$ for every $x_{1} \in J_{1}$. 
Let "." be the Jordan product in $J^{+}$. We define a new multiplication " $x$ " on the set $J$ by $a \times b=a \cdot b-a b$ where $a b$ is the multiplication in $J$. Then $a \cdot b$ $=\frac{1}{2}(a b+b a)$ implies

$$
a \times b=-b \times a
$$

and using the fact that $J$ satisfies the flexible law we obtain

$$
(a \times b) \cdot a+(a \cdot b) \times a=0 .
$$

Linearization of (6) gives

$$
(c \times b) \cdot a+(a \times b) \cdot c+(c \cdot b) \times a+(a \cdot b) \times c=0 .
$$

By Lemma $1(\alpha e)(\beta e)=\left(\bar{L}_{e} \alpha \beta+\bar{R}_{e} \beta \alpha\right) e$, so, from the definition of the $x-$ multiplication, $\alpha e \times \beta e=m(\alpha \beta-\beta \alpha) e$ where $m=\frac{1}{2}-\bar{L}_{e}$.

LEMMA 3. If $\alpha e \in J_{1}$ and $\beta f \in J_{0}$, then $(\alpha e)(\beta f)=0$.

Proof. In (6) with $a=\alpha e, b=\beta f$ we get $(\alpha e \times \beta f) \cdot \alpha e+(\alpha e \cdot \beta f) \times \alpha e=0$. So $(\alpha e \times \beta f) \cdot \alpha e=0$ and if $\alpha e \times \beta f=\xi e+\delta_{12}+\varepsilon f$ where $\delta_{12}=\left(\begin{array}{cc}0 \\ \gamma_{2}^{-1} \delta^{*} & \delta \\ 0\end{array}\right)$, then

$$
\begin{aligned}
0 & \stackrel{\dot{ }}{=}(\alpha e \times \beta b) \cdot \alpha e=\left(\xi e+\delta_{12}+\varepsilon f\right) \cdot \alpha e \\
& =\frac{1}{2}(\alpha \xi+\xi \alpha) e+\frac{1}{2}(\alpha \delta)_{12} .
\end{aligned}
$$

So $\alpha \xi+\xi \alpha=0$ and $\alpha \delta=0$. But $\alpha \in S$ and every nonzero element of $S$ is invertible so $\delta=0$ and $\alpha e \times \beta f$ has zero component in $J_{1 / 2}$. Letting $\alpha=1$ shows $e \times \beta f$ has zero component in $J_{1}$.

Linearization of $(\alpha e \times \beta f) \cdot \alpha e=0$ gives $(\alpha e \times \beta f) \cdot \eta e+(\eta e \times \beta f) \cdot \alpha e=0$. Letting $\eta=1$ we get

$$
(\alpha e \times \beta f) \cdot e+(e \times \beta f) \cdot \alpha e=0
$$

and since the second term of (8) has zero component in $J_{1}(e \times \beta f$ has zero component in $J_{1}$ ) we conclude that $\alpha e \times \beta f$ does also.

With $a=\beta f$ and $b=\alpha e$ in (6) we get $(\beta f \times \alpha e) \cdot \beta f=0$. We recall that $\beta$ has the property that $\gamma_{2}^{-1} \beta^{*} \gamma_{2}=\beta$. Hence $\beta^{*} \gamma_{2}=\gamma_{2} \beta=\left(\beta^{*} \gamma_{2}\right)^{*}$ and so $\gamma_{2} \beta \in S$. This means $\gamma_{2} \beta$ is invertible if $\beta \neq 0$ and we conclude that $\beta$ is invertible also. So now we can repeat the argument used in the first part of the proof of this lemma to get that the $J_{0}$ and $J_{1 / 2}$ components of $\beta f \times \alpha e=-\alpha e \times \beta f$ are zero. Hence $\alpha e \times \beta f=0$. But $\alpha e \times \beta f=\alpha e \cdot \beta f-(\alpha e)(\beta f)$, so $(\alpha e)(\beta f)=0$ and the proof of the lemma is complete.

For notation let $\lambda=\bar{L}_{e}$ and let $\delta_{12}=\left(\begin{array}{ll}0 \\ \gamma \overline{2}^{-1} \delta^{*} & \delta \\ 0\end{array}\right)$. So $\lambda^{*}=\bar{R}_{e}$ and elements of $J_{1 / 2}$ now have the form $\delta_{12}$ where $\delta \in E$.

LEMMA 4. For $\alpha e \in J_{1}$ and $\delta_{12} \in J_{1 / 2},(\alpha e) \delta_{12}=(\lambda \alpha \delta)_{12}$.

Proof. Since $\bar{L}_{x_{1}}, \bar{R}_{x_{1}} \in E$ we have that $J_{1} J_{1 / 2} \subset J_{1 / 2}$ and $J_{1 / 2} J_{1} \subset J_{1 / 2}$, so $\alpha e \times \delta_{12}$ $\in J_{1 / 2}$ for every $\alpha \in S, \delta \in E$. In particular $e \times 1_{12} \in J_{1 / 2}$ so $e \times 1_{12}=n_{12}$ for some $n \in E$. 
Substitution in (7) of $\alpha e, \beta e, \delta_{12}$ for $a, b, c$ respectively gives

$$
\alpha e \cdot\left(\beta e \times \delta_{12}\right)+\frac{1}{2}(m(\beta \alpha-\alpha \beta) \delta)_{12}+\frac{1}{2}(\alpha e) \times(\beta \delta)_{12}=\frac{1}{2}((\alpha \beta+\beta \alpha) e) \times \delta_{12} .
$$

Letting $\alpha=1$ in (9) gives

$$
\frac{1}{2}\left(\beta e \times \delta_{12}\right)+\frac{1}{2} e \times(\beta \delta)_{12}=\beta e \times \delta_{12}, \quad \beta \in S, \delta \in E,
$$

or

$$
\beta e \times \delta_{12}=e \times(\beta \delta)_{12}, \quad \beta \in S, \delta \in E .
$$

With $\beta=1$ in (9) we get

$$
\alpha e \cdot\left(e \times \delta_{12}\right)+\frac{1}{2} \alpha e \times \delta_{12}=\alpha e \times \delta_{12}, \quad \alpha \in S, \delta \in E,
$$

or

$$
2 \alpha e \cdot\left(e \times \delta_{12}\right)=\alpha e \times \delta_{12}, \quad \alpha \in S, \delta \in E .
$$

So for $\delta=1,(10)$ and (11) imply

$$
\begin{aligned}
\alpha e \times 1_{12} & =e \times \alpha_{12}=2 \alpha e \cdot\left(e \times 1_{12}\right) \\
& =2 \alpha e \cdot n_{12}=(\alpha n)_{12}, \quad \alpha \in S .
\end{aligned}
$$

Using (10), (11), and (12) we have for $\alpha, \beta \in S$

$$
\alpha e \times \beta_{12}=e \times(\alpha \beta)_{12}=2 \alpha e \cdot\left(e \times \beta_{12}\right)=2 \alpha e \cdot(\beta n)_{12}=(\alpha \beta n)_{12} .
$$

Now we assume that for any $k$ elements $\alpha_{1}, \alpha_{2}, \ldots, \alpha_{k} \in S$ the following is valid:

$$
\alpha_{k} e \times\left(\alpha_{k-1} \cdots \alpha_{1}\right)_{12}=\left(\alpha_{k} \alpha_{k-1} \cdots \alpha_{1} n\right)_{12} \text {. }
$$

Then for $\alpha_{k+1}, \alpha_{k}, \ldots, \alpha_{1} \in S$ we have

$$
\begin{aligned}
\alpha_{k+1} e \times\left(\alpha_{k} \alpha_{k-1} \cdots \alpha_{1}\right)_{12} & =e \times\left(\alpha_{k+1} \alpha_{k} \cdots \alpha_{1}\right)_{12}=2 \alpha_{k+1} e \cdot\left(e \times\left(\alpha_{k} \cdots \alpha_{1}\right)_{12}\right) \\
& =2 \alpha_{k+1} e \cdot\left(\alpha_{k} \cdots \alpha_{1} n\right)_{12}=\left(\alpha_{k+1} \cdots \alpha_{1} n\right)_{12} .
\end{aligned}
$$

So since $S$ generates $E$, (15) implies that

$$
\alpha e \times \delta_{12}=(\alpha \delta n)_{12}, \quad \alpha \in S, \delta \in E .
$$

Finally, using (16) in (9) and remembering that $E$ is not commutative will show that $m=n=\frac{1}{2}-\bar{L}_{e}=\frac{1}{2}-\lambda$.

The $\times$-multiplication relating $J_{1}$ with $J_{1 / 2}$ is now known so

$$
\begin{aligned}
(\alpha e)\left(\delta_{12}\right) & =(\alpha e) \cdot\left(\delta_{12}\right)-(\alpha e) \times\left(\delta_{12}\right) \\
& =\frac{1}{2}(\alpha \delta)_{12}-\left(\alpha \delta\left(\frac{1}{2}-\lambda\right)\right)_{12} \\
& =\frac{1}{2}(\alpha \delta)_{12}-\frac{1}{2}(\alpha \delta)_{12}+(\alpha \delta \lambda)_{12}=(\lambda \alpha \delta)_{12} .
\end{aligned}
$$

Lemma 5. If $\delta_{12}, \eta_{12} \in J_{1 / 2}$, then the $J_{0}$-component of $\delta_{12} \eta_{12}$ is

$$
\left(\lambda \gamma_{2}^{-1} \delta^{*} \eta+\lambda^{*} \gamma_{2}^{-1} \eta^{*} \delta\right) f .
$$


Proof. In (7) let $a=e, b=\eta_{12}$ and $c=\delta_{12}$ to get

$$
\left(\delta_{12} \times \eta_{12}\right) \cdot e+\left(e \times \eta_{12}\right) \cdot \delta_{12}+\left(\delta_{12} \cdot \eta_{12}\right) \times e+\left(e \cdot \eta_{12}\right) \times \delta_{12}=0 .
$$

Since $\delta_{12} \cdot \eta_{12} \in J_{1}+J_{0}$ the third term of (17) is zero using Lemmas 1 and 3. Also, $e \times \eta_{12}=e \cdot \eta_{12}-e \eta_{12}=\frac{1}{2} \eta_{12}-\lambda \eta_{12}=\left(\frac{1}{2}-\lambda\right) \eta_{12}$ using Lemma 4. So (17) reduces to

$$
\left(\delta_{12} \times \eta_{12}\right) \cdot e+\left(\left(\frac{1}{2}-\lambda\right) \eta\right)_{12} \cdot \delta_{12}+\frac{1}{2} \eta_{12} \times \delta_{12}=0 .
$$

$\left(\delta_{12} \times \eta_{12}\right) \cdot e$ has zero component in $J_{0}$ so (18) says

$$
\left[\delta_{12} \times \eta_{12}\right]_{0}=\left[\eta_{12} \cdot \delta_{12}\right]_{0}-\left[(2 \lambda \eta)_{12} \cdot \delta_{12}\right]_{0} .
$$

But

$$
\begin{aligned}
{\left[\delta_{12} \eta_{12}\right]_{0} } & =\left[\delta_{12} \cdot \eta_{12}\right]_{0}-\left[\delta_{12} \times \eta_{12}\right]_{0}=\left[(2 \lambda \eta)_{12} \cdot \delta_{12}\right]_{0} \\
& =\left(\gamma_{2}^{-1} \delta^{*} \lambda \eta+\gamma_{2}^{-1}(\lambda \eta)^{*} \delta\right) f=\left(\lambda \gamma_{2}^{-1} \delta^{*} \eta+\lambda^{*} \gamma_{2}^{-1} \eta^{*} \delta\right) f .
\end{aligned}
$$

Lemma 6. If $\delta_{12}, \eta_{12} \in J_{1 / 2}$, then the $J_{1 / 2}$-component of $\delta_{12} \eta_{12}$ is 0 .

Proof. In (7) let $a=\alpha e, b=\eta_{12}, c=\delta_{12}$, and comparing components in $J_{1 / 2}$ will give

$$
2\left[\left(\delta_{12} \times \eta_{12}\right) \cdot \alpha e\right]_{1 / 2}=\left[\delta_{12} \times(\alpha \eta)_{12}\right]_{1 / 2}, \quad \alpha \in S, \delta, \eta \in E .
$$

Again in (7) let $c=\delta_{12}, b=\alpha e, a=\eta_{12}$ and get

$$
(\alpha \delta)_{12} \times \eta_{12}=\delta_{12} \times(\alpha \eta)_{12}, \quad \alpha \in S, \delta, \eta \in E .
$$

For $\alpha \in S$ we have from (19) that $\left[1_{12} \times \alpha_{12}\right]_{1 / 2}=2\left[\left(1_{12} \times 1_{12}\right) \cdot \alpha e\right]_{1 / 2}=0$. For $\alpha, \beta \in S$, (20) and (19) say $\left[\beta_{12} \times \alpha_{12}\right]_{1 / 2}=\left[1_{12} \times(\beta \alpha)_{12}\right]_{1 / 2}=2\left[\left(1_{12} \times \alpha_{12}\right) \cdot \beta e\right]_{1 / 2}=0$. Using an inductive process as in Lemma 4 and recalling that $S$ generates $E$ will give $\left[\delta_{12} \times \eta_{12}\right]_{1 / 2}=0$ for every $\delta, \eta \in E$. This implies $\left[\delta_{12} \eta_{12}\right]_{1 / 2}=0$.

3. The first structure theorem. With the aid of the lemmas it is now quite easy to prove our first theorem.

THEOREM 1. Let $J$ be a simple noncommutative Jordan algebra of capacity two such that $\mathrm{J}^{+}$is not a Jordan algebra determined by a symmetric bilinear form. Then $J$ is either commutative Jordan or quasi-associative.

Proof. Let "。" denote ordinary associative matrix multiplication in $E_{2}$. If $x_{1}, y_{1} \in J_{1}$, Lemma 1 says $x_{1} y_{1}=\lambda \circ x_{1} \circ y_{1}+(1-\lambda) \circ y_{1} \circ x_{1}$. If $x_{1} \in J_{1}, y_{1 / 2} \in J_{1 / 2}$, Lemma 4 says $x_{1} y_{1 / 2}=\lambda \circ x_{1} \circ y_{1 / 2}+(1-\lambda) \circ y_{1 / 2} \circ x_{1}$.

Since $f \delta_{12}=(1-e) \delta_{12}=\delta_{12}-e \delta_{12}=\delta_{12}-(\lambda \delta)_{12}=((1-\lambda) \delta)_{12}=\delta_{12} e$ we have that $\bar{L}_{f}=(1-\lambda)=\lambda^{*}=\bar{R}_{e}$. Symmetry of the arguments used in the lemmas with respect to $f$ will give corresponding multiplications with $J_{0}$ and the analogue of Lemma 5 together with Lemma 6 will show that $\delta_{12} \eta_{12}=\lambda \circ \delta_{12} \circ \eta_{12}+(1-\lambda) \circ \eta_{12} \circ \delta_{12}$. If $\lambda=\lambda^{*}, J$ is commutative Jordan and if $\lambda \neq \lambda^{*}, J$ is quasi-associative.

If $E=\Delta \oplus \bar{\Delta}$, a direct sum of a division ring $\Delta$ with its anti-isomorphic copy $\bar{\Delta}$, then $J$ is an algebra over $\Phi=\{(\alpha, \bar{\alpha}) \mid \alpha \in$ center of $\Delta\}$. Any skew element $\lambda$ in 
$\Delta \oplus \bar{\Delta}$ is of the form $\lambda=(\beta,-\bar{\beta})$. Hence if $\lambda$ is skew and central then $\lambda^{2}=(\beta,-\bar{\beta})^{2}$ $=\left(\beta^{2}, \bar{\beta}^{2}\right)$ which has a square root in $\Phi$. So by a theorem of McCrimmon [3, p. 1458], $J$ is split quasi-associative.

If $E$ is a division ring with a central skew element $\lambda$ then $\lambda^{2}$ does not have a square root in $\Phi$, the basefield of $E$. Hence by McCrimmon's theorem, $J$ is nonsplit quasi-associative.

4. The second structure theorem. For the rest of this work we will assume $J$ is a noncommutative Jordan algebra such that $J^{+}$is a Jordan algebra determined by a symmetric bilinear form. Then $J^{+}=\Phi+V$ where $\Phi$ is a field, $V$ is a vector space over $\Phi$, and $f: V \times V \rightarrow \Phi$ is a symmetric bilinear form on $V$. If we denote the elements of $J^{+}$by $(\alpha, v)$ where $\alpha \in \Phi, v \in V$ then the multiplication in $J^{+}$is given by $(\alpha, v)(\beta, w)=(\alpha \beta+f(v, w), \alpha w+\beta v)$. It can easily be shown that if $f$ is nondegenerate, then $J^{+}$is simple. The elements of $J$ will also be denoted by ordered pairs of elements from $\Phi$ and $V$. If $J^{+}$has an idempotent $e \neq(1,0)$, then $J^{+}$has capacity two. An easy calculation will show that such an idempotent exists if and only if $f(u, u)=\frac{1}{4}$ for some vector $u \in V$.

If $f$ is not identically 0 there exists an element $w \in V$ such that $f(w, w)=\beta \neq 0$. If $\alpha=\beta^{1 / 2} \in \Phi$, then $f\left(\frac{1}{2} \alpha^{-1} w, \frac{1}{2} \alpha^{-1} w_{\delta}\right)=\frac{1}{4}$ and $J^{+}$(and $J$ ) contains a nontrivial idempotent. If $\beta^{1 / 2} \notin \Phi$ we may take a quadratic extension of $\Phi$ to obtain an idempotent. So after a possible quadratic extension of the base field $\Phi$ we may assume $J$ has capacity two.

Henceforth $u$ will denote the vector in $V$ such that $f(u, u)=\frac{1}{4}$. Then $e=\left(\frac{1}{2}, u\right)$ and $f=\left(\frac{1}{2},-u\right)$ are two orthogonal idempotents such that $1=e+f$. With respect to $e$ we have

$$
\begin{aligned}
J_{0} & =\left\{\left(\frac{1}{2} \alpha,-\alpha u\right) \mid \alpha \in \Phi\right\}, \\
J_{1 / 2} & =\{(0, w) \mid f(u, w)=0\}, \\
J_{1} & =\left\{\left(\frac{1}{2} \alpha, \alpha u\right) \mid \alpha \in \Phi\right\} .
\end{aligned}
$$

We recall that $E$ is the associative ring generated by $\left\{2 \bar{R}_{\alpha e}^{+} \mid \alpha e=\left(\frac{1}{2} \alpha, \alpha u\right) \in J_{1}\right\}$. Since $\bar{R}_{\alpha e}^{+} \bar{R}_{\beta e}^{+}=\bar{R}_{\beta e}^{+} \bar{R}_{\alpha e}^{+}, E$ is a field isomorphic to $\Phi$. Since $\nu\left(x_{1}\right) \nu\left(y_{1}\right)=\nu\left(y_{1}\right) \nu\left(x_{1}\right)$, equation (3) says that $\nu\left(x_{1} y_{1}\right)=v\left(x_{1}\right) \nu\left(y_{1}\right)$. So $J_{1}$ is a field isomorphic to $\Phi$.

We will have occasions to identify the element $(0, v)$ with the vector $v \in V$, so we will make this identification whenever necessary. $J$ induces a multiplication on the vectors of $V$ as follows: for $w, y \in V$ define

$$
w \times y=[(0, w)(0, y)]_{1 / 2},
$$

i.e. $w \times y$ is the $J_{1 / 2}$-component of the product $(0, w)(0, y)$ in $J$. Clearly we have

$$
w \times y=-y \times w .
$$

For $a_{1 / 2}=(0, w) \in J_{1 / 2}$ we know $e a_{1 / 2} \in J_{1 / 2}$, so $e(0, w)=\left(\frac{1}{2}, u\right)(0, w)=\left(\frac{1}{2}, 0\right)(0, w)$ $+(0, u)(0, w)=\left(0, \frac{1}{2} w\right)+(0, u)(0, w)$. Hence $(0, u)(0, w) \in J_{1 / 2}$, so $(0, u)(0, w)=$ $(0, u \times w)$. 
Lemma 7. If $\alpha e \in J_{1}$ and $(0, w) \in J_{1 / 2}$, then $(\alpha e)(0, w)=\left(0, \frac{1}{2} \alpha w+\alpha(u \times w)\right)$.

Proof. The linearized flexible law says

$$
(\alpha e)[e(0, w)]+(0, w)[e(\alpha e)]=[(\alpha e) e](0, w)+[(0, w) e](\alpha e)
$$

or

$$
(\alpha e)\left(0, \frac{1}{2} w+u \times w\right)+(0, w)(\alpha e)=(\alpha e)(0, w)+\left(0, \frac{1}{2} w+w \times u\right)(\alpha e) .
$$

Due to the fact that we know the multiplication in $J^{+},(22)$ becomes

$$
\begin{aligned}
(\alpha e)\left(0, \frac{1}{2} w+u \times w\right)+ & (0, \alpha w)-(\alpha e)(0, w) \\
& =(\alpha e)(0, w)+\left(0, \frac{1}{2} \alpha w+\alpha(w \times u)\right)-(\alpha e)\left(0, \frac{1}{2} w+w \times u\right) .
\end{aligned}
$$

So $(\alpha e)(0, w)=\left(0, \frac{1}{2} \alpha w+\alpha(u \times w)\right)$.

LEMMA 8. Let $v, w \in V$, then $(0, v)(0, w)=(f(v, w), v \times w)$.

Proof. The flexible law says

$$
(0, v)[(0, w)(0, v)]-[(0, v)(0, w)](0, v)=0,
$$

and so we have

$$
(0, v)[(0, w)(0, v)]+[(0, w)(0, v)](0, v)=2(f(v, w), 0)(0, v) .
$$

Hence we know that the product in $J^{+}$of $(0, v)$ with $(0, w)(0, v)$ is the same as that of $(0, v)$ with $(f(v, w), 0)$. This together with $(21)$ implies that $(0, v)(0, w)$ $=(f(v, w), v \times w)$.

Lemmas 7 and 8 imply the following theorem which was motivated by Theorem 1 of [7].

THEOREM 2. If $J$ is a noncommutative Jordan algebra such that $J^{+}=\Phi+V$ is a Jordan algebra determined by a nonzero symmetric bilinear form, then possibly after a quadratic extension of the field $\Phi$ the multiplication in $J$ is given by

$$
(\alpha, v)(\beta, w)=(\alpha \beta+f(v, w), \alpha w+\beta v+v \times w)
$$

where " $x$ " is an anti-commutative multiplication on the vector space $V$ and $f$ is the symmetric bilinear form of $J^{+}$such that $f(v \times w, y)=f(v, w \times y)$.

If we assume that $V$ has a basis $\left\{w_{i}\right\}$ over $\Phi$ such that $f\left(w_{i}, w_{j}\right)=0$ if $i \neq j$ and $f\left(w_{i}, w_{i}\right)=\beta_{i}$, then we can give more information about the $\times$-multiplication on $V$. Let $w_{i} \times w_{j}=\sum_{k} \gamma_{i j k} w_{k}$ where $\gamma_{i j k} \in \Phi$. Then $w_{i} \times w_{j}=-w_{j} \times w_{i}$ means that

$$
\gamma_{i j k}=-\gamma_{j i k} \text {. }
$$

Also $f\left(w_{i}, w_{j} \times w_{k}\right)=f\left(w_{i} \times w_{j}, w_{k}\right)$ is equivalent to

$$
f\left(w_{k}, w_{k}\right) \gamma_{i j k}=f\left(w_{i}, w_{i}\right) \gamma_{j k i}=f\left(w_{j}, w_{j}\right) \gamma_{k i j} .
$$

So given any set of constants $\left\{\gamma_{i j k}\right\}$ satisfying (23) and (24), $w_{i} \times w_{j}=\sum_{k} \gamma_{i j k} w_{k}$ gives a multiplication on $V$ such that $J$ is a noncommutative Jordan algebra and $J^{+}$is a 
Jordan algebra determined by a symmetric bilinear form. Whenever $V$ has an orthogonal basis, such a set of $\gamma_{i j k}$ 's can always be found, for we can define $\gamma_{i j k}$ arbitrarily for $i<j<k$ and then use (23) and (24) to find $\gamma_{i j k}$ for the other permutations of $i, j, k$.

\section{REFERENCES}

1. N. Jacobson, Structure and representations of Jordan algebras, Amer. Math. Soc. Colloq. Publ., vol. 39, Amer. Math. Soc., Providence, R. I., 1968. MR 40 \#4330.

2. - Structure theory for a class of Jordan algebras, Proc. Nat. Acad. Sci. U.S.A. 55 (1966), 231-251. MR 33 \#1337.

3. K. McCrimmon, A note on quasi-associative algebras, Proc. Amer. Math. Soc. 17 (1966), 1455-1459. MR 39 \#270.

4. - Structure and representations of noncommutative Jordan algebras, Trans. Amer. Math. Soc. 121 (1966), 187-199. MR 32 \#5700.

5. K. McCrimmon and R. D. Schafer, On a class of noncommutative Jordan algebras, Proc. Nat. Acad. Sci. U.S.A. 56 (1966), 1-4. MR 34 \#5888.

6. J. M. Osborn, Jordan algebras of capacity two, Proc. Nat. Acad. Sci. U.S.A. 57 (1967), 582-588. MR 35 \#6727.

7. — Quadratic division algebras, Trans. Amer. Math. Soc. 105 (1962), 202-221. MR 25 \#3968.

UNIVERSITY OF MISSISSIPPI,

UNIVERSITY, MISSISSIPPI 38677 